\title{
Linfadenectomía lumboaórtica asistida por robot en tumor residual postquimioterapia en cáncer testicular*
}

\author{
Drs. OCTAVIO A. CASTILLO C. ${ }^{1,2,3}$, FRANCISCO SEPÚLVEDA T. ${ }^{1}$, \\ LUIS F. SUSANIBAR N. ${ }^{1}$, IVAR VIDAL M. ${ }^{1}$, GONZALO RUBIO L. ${ }^{1}$ \\ 1 Unidad de Urología y Centro de Cirugía Robótica, Clínica Indisa. \\ 2 Facultad de Medicina, Universidad Andrés Bello. \\ 3 Facultad de Medicina Universidad de Chile. \\ Santiago, Chile.
}

\begin{abstract}
Robotic-assisted laparoscopic retroperitoneal lymph node dissection in post chemotherapy residual mass in testis cancer

Introduction: The laparoscopic retroperitoneal lymph node dissection (L-RPLND) has shown results at least comparable to open surgery in terms of perioperative complications and oncological results, but its application in the post chemotherapy scenario is still matter of study. The development of robotic surgery and its advantages over laparoscopic surgery, make this an attractive option for complex procedures. We report our initial experience with robotic-assisted retroperitoneal lymph node dissection (R-RPLND). Methods: We describe the cases of two patients who underwent R-RPLND due to a Post Chemotherapy residual mass of a non-seminomatous testicular cancer. Results: Two patients, 27 and 30 years old, presented with retroperitoneal residual mass after 4 and 6 cycles of Bleomicin, Etoposide and Cis-Platinum. The first patient had a 4.3 $\mathrm{cm}$ left para-aortic mass and the other had a $6 \mathrm{~cm}$ mass behind the third portion of the duodenum. The mean surgical time was 255 minutes (210-300), with an estimated mean blood loss of 450 cc (100-800) and a mean hospital stay of 60 hours (72-48). The pathologic result was Teratoma in both cases. There was no perioperative morbidity. Conclusions: We believe that R-RPLND is a feasible and safe alternative in selected patients. However still needs more experience and longer follow up to asess its oncological outcome.
\end{abstract}

Key words: Robotic surgery, retroperitoneal lymph node dissection, testis cancer.

\section{Resumen}

Introducción: La linfadenectomía retroperitoneal lumboaórtica laparoscópica (LRLA-L) ha demostrado resultados al menos comparables a la cirugía abierta en términos de complicaciones peri operatorias y resultados oncológicos. Sin embargo, su aplicación en el escenario postquimioterapia es todavía materia de

*Recibido el 17 de agosto de 2010 y aceptado para publicación el 12 de enero de 2011.

Correspondencia: Dr. Octavio A. Castillo C.

Av. Apoquindo 3990, Of. 809, Santiago, Chile. Fax: (56-2) 228 4269. C.P.: 7550112

octavio.castillo@indisa.cl 
estudio. El desarrollo de la cirugía robótica y sus ventajas sobre la cirugía laparoscópica hacen de este tipo de cirugía una opción atractiva para procedimientos más complejos como esta intervención postquimioterapia. Reportamos nuestra experiencia inicial en Linfadenectomía lumboaórtica laparoscópica robóticamente asistida postquimioterapia. Métodos: Describimos el caso de dos pacientes sometidos a una a una linfadenectomía retroperitoneal lumboaórtica asistida por robot (LRLA-R), portadores de una masa retroperitoneal postquimioterapia secundaria a un carcinoma testicular de células germinales no seminoma. Resultados: Dos pacientes, de 27 y 30 años de edad, con una masa retroperitoneal residual después de 4 y 6 ciclos de Bleomicina, Etoposido y Cis-Platinum respectivamente. El primer paciente presentó una masa para-aórtica izquierda de 4,3 cm y el segundo paciente una masa en relación a la tercera porción del duodeno de $6 \mathrm{~cm}$. El tiempo operatorio promedio fue de 255 minutos (210-300) con un sangrado promedio estimado de $450 \mathrm{ml}$ (100-800). El tiempo promedio de hospitalización fue de 60 hrs (48-72). En ambos casos el resultado histológico evidenció la presencia de Teratoma. No se presentaron complicaciones perioperatorias. Conclusiones: Creemos que la LRLA-R en pacientes con masa residual post-quimioterapia es una técnica reproducible y segura en pacientes seleccionados, sin embargo, todavía es necesario un mayor número de pacientes y tiempos de seguimiento para poder evaluar los resultados oncológicos.

Palabras clave: Cirugía robótica, Linfadenectomía Lumboaórtica, cáncer de testículo.

\section{Introducción}

La linfadenectomía retroperitoneal lumboaórtica abierta (LRLA-A) es el método de elección en muchos centros para el manejo de pacientes con tumores testiculares de células germinales no seminoma. Sin embargo, el desarrollo de la cirugía laparoscópica en los últimos años ha convertido a esta última en una alternativa al menos comparable a la cirugía abierta para el manejo de estos pacientes ${ }^{1-4}$. No obstante, en aquellos pacientes que deben ser sometidos a una linfadenectomía retroperitoneal lumboaórtica (LRLA) post quimioterapia, el escenario es completamente distinto. Si bien diversos autores describen tiempos de hospitalización y control de la enfermedad comparables a la cirugía abierta, su uso sólo es recomendado en centros con importante experiencia en cirugía laparoscópica ${ }^{5-10}$.

La cirugía laparoscópica asistida por robot (da Vinci System, Intuitive) ha sido descrita como vía de abordaje en múltiples procedimientos urológicos como la prostatectomía radical, adrenalectomía, nefrectomía radical y parcial, demostrando resultados comparables a la cirugía abierta y laparoscópica. $\mathrm{Al}$ agregar una visión tridimensional, un mayor rango de movimiento y la ausencia de temblor, la cirugía robóticamente asistida se convierte en una opción atractiva para procedimientos de mayor complejidad como la linfadenectomía retroperitoneal lumboaórtica en pacientes con masa residual postquimioterapia.

Presentamos 2 casos clínicos de pacientes sometidos a una LRLA-R por masa residual retroperitoneal luego de tratamiento quimioterápico por cáncer testicular avanzado, que hasta donde sabemos son los primeros casos reportados en Latinoamérica y el $4^{\circ}$ y $5^{\circ}$ reportados en la literatura mundial.

\section{Casos clínicos}

\section{Caso 1}

Paciente varón de 30 años, sin antecedentes mórbidos, quien tiene el antecedente de una orquiectomía radical derecha cuyo estudio anatomopatológico revela un Carcinoma Embrionario + Teratoma inmaduro (teratocarcinoma) con mínimos focos de seminoma moderadamente diferenciado Grado IV de Dixon y Moore.

Tras la orquiectomía los marcadores se tornan negativos manteniéndose desde entonces con vigilancia estrecha mediante exploración física, radiografía de tórax, TAC y marcadores tumorales cada 3 meses.

Exactamente un año después hay aumento significativo de marcadores tumorales y la tomografía computada muestra una masa retroperitoneal intercavoaórtica, en relación con la $3{ }^{a}$ porción del duodeno asociada a una imagen compatible con trombosis de vena cava, sin fenómenos de tromboembolismo. El paciente es tratado con terapia anticoagulante oral (Coumadin) y se inicia qumioterapia con Bleomicina, Etoposido y Cisplatino (BEP), completando 6 ciclos. Es derivado a nuestra clínica por persistencia de la masa retroperitoneal de $4 \mathrm{~cm}$ de diámetro (Figura 1) y marcadores negativos, para considerar cirugía complementaria. En diciembre de 2009 se realiza LRLA-R derecha (Figuras 2 y 3), siguiendo la técnica previamente descrita para acceso laparoscópico ${ }^{4}$.

La cirugía tuvo una duración de 300 minutos (5 horas) con sangrado estimado de $800 \mathrm{ml}$. No fue necesario el uso de narcóticos para analgesia ni se requirió transfusión. El paciente fue dado de alta antes de 72 horas.

La biopsia informó una masa retroperitoneal de $6 \mathrm{~cm}$ con componente viable de Teratoma maduro y 15 linfonodos sin metástasis. 


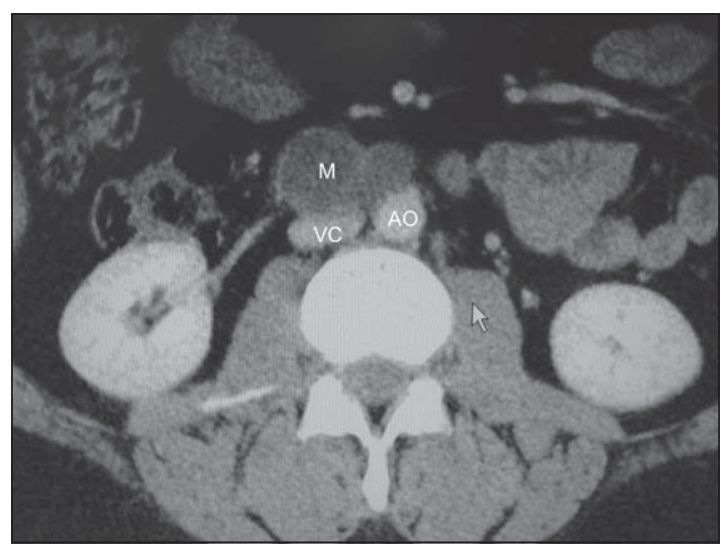

Figura 1. Masa retroperitoneal derecha: M: masa, VC: vena cava, $\mathrm{AO}$ : Aorta.

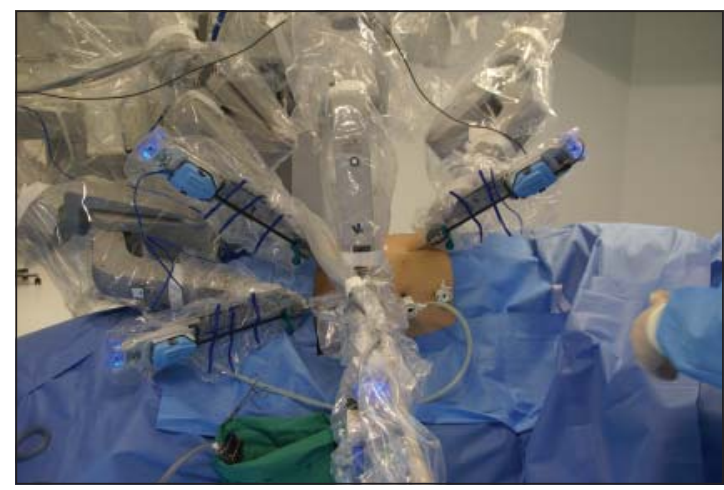

Figura 3. Posición de los brazos robóticos (4) para linfadenectomía retroperitoneal derecha.

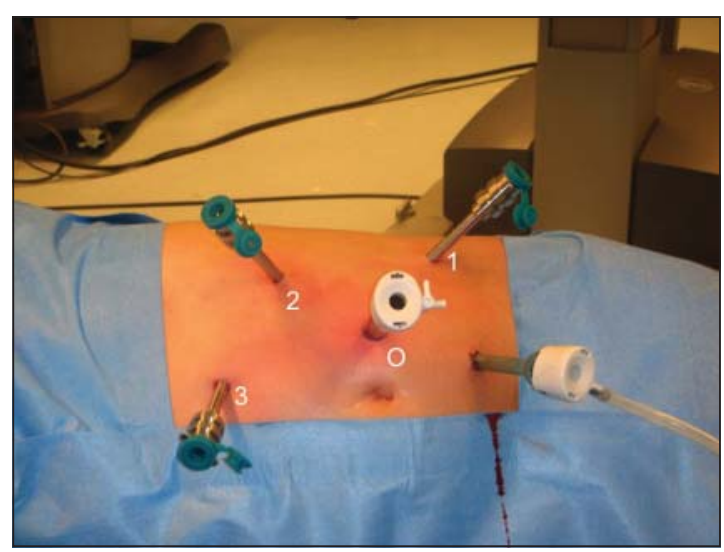

Figura 5. Puertos para cirugía retroperitoneal robótica izquierda. Marcado con 0 es el puerto para la óptica, y los puertos 1, 2 y 3 son para los instrumentos robóticos. El trocar no marcado es para uso del ayudante.

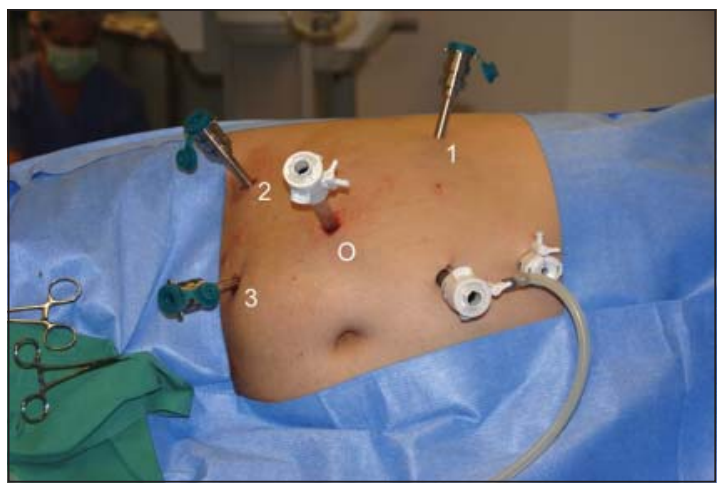

Figura 2. Puertos para cirugía retroperitoneal robótica derecha. Marcado con 0 es el puerto para la óptica, y los puertos 1, 2 y 3 son para los instrumentos robóticos. El trocar no marcado de $10 \mathrm{~mm}$ es para uso del ayudante y el trocar de $5 \mathrm{~mm}$ a su lado es para desplazar el hígado.

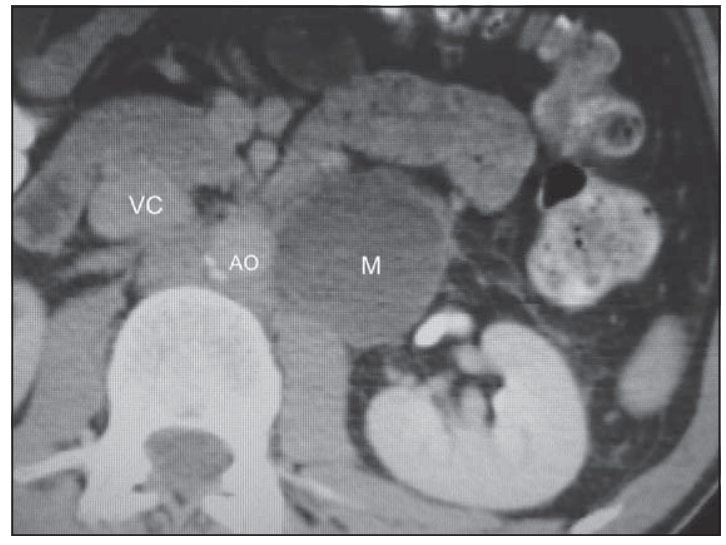

Figura 4. Masa paraaórtica retroperitoneal izquierda. M: masa, AO: aorta, VC: vena cava.

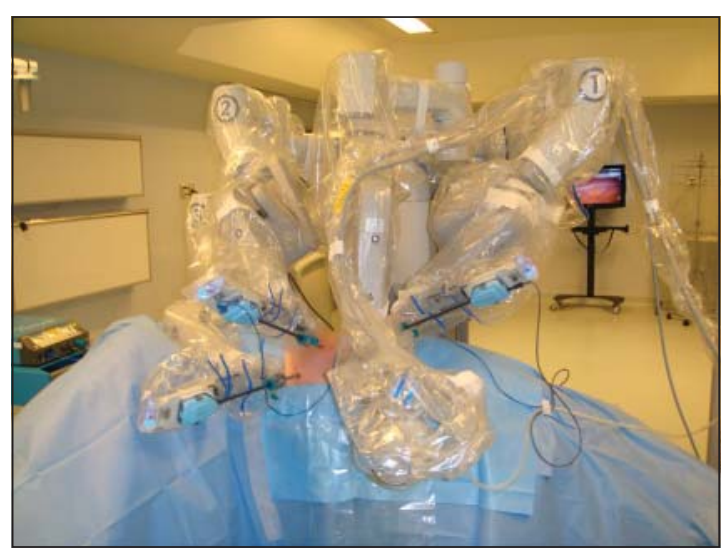

Figura 6. Posición de los brazos robóticos (4) para linfadenectomía retroperitoneal izquierda. 
Seis meses después el paciente se encuentra sin evidencia de enfermedad y con eyaculación anterógrada.

\section{Caso 2}

Paciente de 27 años, con el antecedente de haber sido operado por un tumor testicular izquierdo cuya biopsia fue informada como Carcinoma embrionario más teratoma. Al momento del diagnóstico sus exámenes radiológicos mostraban metástasis pulmonares y retroperitoneales.

Se realizó quimioterapia con esquema clásico (BEP) en 3 ciclos con desaparición completa de las lesiones pulmonares. Por persistencia de una masa retroperitoneal para aórtica izquierda, de $2,5 \mathrm{~cm}$ en la tomografía computada (Figura 4), el paciente consultó en nuestro centro para evaluar la posibilidad de cirugía mínimamente invasiva.

En marzo de 2010 se realizó una linfadenectomía retroperitoneal LRLA-R (Figuras 5 y 6). El tiempo quirúrgico fue de 210 minutos, con un sangrado estimado de $100 \mathrm{ml}$. El paciente no requirió analgesia con opiáceos y fue dado de alta antes de 48 horas. La biopsia fue informada como masa retroperitoneal de 4,3 cm compatible con Teratoma viable y 18 linfonodos con 9 de ellos con metástasis de Teratoma con involución completa.

El paciente está asintomático a 4 meses de la cirugía, aun cuando no ha presentado eyaculación anterógrada

\section{Discusión}

El desarrollo de la quimioterapia basada en Cisplatino, sumado al gran avance imagenológico de los últimos años, han incrementado significativamente la sobrevida en los pacientes con tumores testiculares de células germinales en estadios avanzados ${ }^{2}$. No obstante en aquellos pacientes que, sometidos a un tratamiento quimioterapéutico, presentan un tumor residual y normalización de los marcadores tumorales, es la linfadenectomía lumboaórtica la que juega un rol protagónico. Sin embargo, debido a la gran reacción desmoplástica que produce la quimioterapia en los tejidos, la linfadenectomía retroperitoneal es una de las cirugías más complejas a las que puede verse expuesto el cirujano urólogo.

Dado que la gran mayoría de estos tumores residuales contendrán sólo fibrosis o necrosis, algunos autores han postulado diversos criterios para identificar a aquellos pacientes que no se beneficiaran de una cirugía (TAC normal, tumor menor de 1,5 cm o menor de $20 \mathrm{ml}, 90 \%$ de reducción de la masa tumoral y ausencia de teratoma en el tumor testicular $)^{7,8}$. Sin embargo, ninguno de ellos ha podido ser validado para identificar a aquellos tumores que sólo contendrán necrosis, por lo que la linfadenectomía retroperitoneal lumboaórtica constituye la opción terapéutica de elección en estos pacientes ${ }^{7}$.

La linfadenectomía retroperitoneal abierta en masa residual post quimioterapia se asocia con una tasa de morbilidad y mortalidad de $20 \%$ y $0,8 \%{ }^{11}$ respectivamente, por lo que en un intento por disminuir estos valores, múltiples autores han postulado la cirugía laparoscópica como vía inicial de abordaje $\mathrm{e}^{5-10}$.

Rassweiler et $\mathrm{al}^{12}$, describe en una serie de 9 pacientes abordados por vía laparoscópica con una tasa de conversión de $67 \%$, concluyendo que este tipo de abordaje no es recomendable, principalmente producto de las grandes dificultades en la disección y las consecuentes complicaciones vasculares. Sin embargo, Albqami et al $^{13}$, en una serie de 59 pacientes en estadio II (43 pacientes en estadio IIB y 16 pacientes en estadio IIC) sometidos a una LRLA-L postquimioterapia, sólo describe complicaciones vasculares menores capaces de ser controladas laparoscópicamente. En ningún caso se requirió conversión a cirugía abierta. Se describe una recidiva tumoral a los 24 meses en un paciente en estadio IIB, la que se encontró fuera de los márgenes quirúrgicos y fue resecada laparoscópicamente. El estudio anatomopatológico final reveló la presencia de Teratoma maduro.

Por otro lado, Maldonado et $\mathrm{al}^{5}$, en una serie de 17 pacientes en estadios II-III con tumores mayores a $1 \mathrm{~cm}$, tampoco describe complicaciones perioperatorias ni necesidad de conversión a cirugía abierta. Ambos autores concluyen que, en manos expertas, la LRLA-L en masa postquimioterapia es una técnica reproducible y segura, no obstante, reconocen que sus tiempos de seguimiento son aún insuficientes para evaluar los resultados oncológicos.

En tiempos de cirugía mínimamente invasiva la cirugía robóticamente asistida no es la excepción. Dadas las ventajas que esta vía de abordaje ofrece a la cirugía laparoscópica, este tipo de técnica se presenta como una alternativa atractiva para cirugías de gran complejidad como lo es la disección retroperitoneal en masa residual post qumioterapia.

En el año 2006 Davol et al ${ }^{14}$, describen la primera LRLA-R en un paciente con un carcinoma de células germinales no seminoma etapa I. El procedimiento fue llevado a cabo sin incidentes siendo el paciente dado de alta a las 48 hrs. Sin embargo, fue el año 2008 cuando Annerstedt et $\mathrm{al}^{15}$, describen los primeros casos de LRLA-R en un paciente en estadio IIA y dos pacientes en estadio IIB. En los 3 casos los pacientes fueron sometidos a ciclos de BEP (Bleomicina, Etopósido y Cisplatino) producto de un carcinoma de células germinales no seminomatoso. En 
los 3 casos los pacientes persistieron con un tumor retroperitoneal residual motivo por el que se realizó la LRLA-R. En todos los casos el procedimiento fue finalizado con éxito, sin complicaciones intraoperatorias ni postoperatorias siendo todos los pacientes dados de alta a las 56 horas promedio (48-72 horas).

Presentamos nuestra experiencia en dos pacientes sometidos a una LRLA-R que hasta donde sabemos se trata de los primeros casos reportados en Latinoamérica y cuarto y quinto casos en el mundo. $\mathrm{Al}$ igual que las series descritas anteriormente los procedimientos fueron llevados a cabo sin complicaciones perioperatorias, siendo ambos pacientes dados de alta en buenas condiciones generales en promedio a las 60 hrs post cirugía.

Al ser comparada con la cirugía laparoscópica convencional, la cirugía robótica nos ofrece una mayor facilidad en la disección, principalmente gracias a su mayor rango de movimiento y ausencia de temblor. Por otra parte, los costos de implementación de la cirugía robótica son compensados al requerir una menor dosis de analgésicos postoperatorios y un menor tiempo de hospitalización.

\section{Conclusión}

Creemos que la LRLA-R es una técnica reproducible y segura en pacientes seleccionados. Sin embargo, todavía es necesario un mayor número de pacientes y tiempos de seguimiento para poder evaluar los resultados oncológicos a largo plazo.

\section{Referencias}

1. Rassweiler JJ, Scheitlin W, Heidenreich A, Laguna MP, Janetschek G. Laparoscopic retroperitoneal lymph node dissection: does it still have a role in the management of clinical stage I nonseminomatous testis cancer? A European perspective. Eur Urol. 2008;54:1004-15.

2. Steiner H, Peschel R, Bartsch G. Laparoscopic retroperitoneal lymph node dissection: current concepts and limitations. BJU Int. 2009;104(9 Pt B):1376-80.

3. Janetschek G, Peschel R, Hobisch A, Bartsch G. Laparoscopic retroperitoneal lymph node dissection. J
Endourol. 2001;15:449-53,discussion 453-5.

4. Castillo O, Rioja J, Vidal-Mora I, Feria M, SánchezSalas R. Morbilidad de la linfadenectomía lumboaórtica laparoscópica en pacientes con tumor testicular no seminomatoso en estadio clínico A. Rev. Chilena de Cirugía 2009;61:533-7.

5. Maldonado-Valadez R, Schilling D, Anastasiadis AG, Sturm W, Stenzl A, Corvin S. Post-chemotherapy laparoscopic retroperitoneal lymph-node dissection in testis cancer patients. J Endourol. 2007;21:1501-4.

6. Palese MA, Su LM, Kavoussi LR. Laparoscopic retroperitoneal lymph node dissection after chemotherapy. Urology 2002;60:130-4.

7. Winter C, Raman JD, Sheinfeld J, Albers P. Retroperitoneal lymph node dissection after chemotherapy. BJU Int. 2009;104(9 Pt B):1404-12.

8. Heidenreich A, Thüer D, Polyakov S. Postchemotherapy retroperitoneal lymph node dissection in advanced germ cell tumours of the testis. Eur Urol. 2008;53:260-74.

9. Heidenreich A, Pfister D, Witthuhn R, Thüer D, Albers P. Postchemotherapy retroperitoneal lymph node dissection in advanced testicular cancer: radical or modified template resection. Eur Urol. 2009;55:217-24.

10. Permpongkosol S, Lima GC, Warlick CA, Allaf ME, Varkarakis IM, Bagga HS, et al. Postchemotherapy laparoscopic retroperitoneal lymph node dissection: evaluation of complications. Urol. 2007;69:361-5.

11. Baniel J, Foster R, Rowland R. Testis cancer: complications of post-chemotherapy retroperitoneal lymph node dissection. J Urol. 1995;153:976-980.

12.- Rassweiler JJ, Seemann O, Henkel TO, Stock C, Frede T, Alken P. Laparoscopic retroperitoneal lymph node dissection for nonseminomatous germ cell tumors: Indications and limitations. J Urol. 1996;156:1108-13.

13. Albqami N, Janetschek G. Laparoscopic Retroperitoneal Lymph-Node Dissection in the Management of Clinical Stage I and II Testicular Cancer. J Endourol. 2005;19:683-92.

14. Davol P, Sumfest J, Rukstalis D. Robotic-assisted laparoscopic retroperitoneal lymph node dissection. Urology 2006;67:199.

15. Annerstedt M, Gudjonsson S, Wullt B, Uvelius B. Robot-assisted laparoscopic retroperitoneal lymph node dissection in clinical stage II testicular cancer. J Robotic Surg. 2008;2:189-91. 